\title{
Neurobehavioral and neurodevelopmental effects of pesticide exposures
}

\section{Citation}

London, Leslie, Cheryl Beseler, Maryse F. Bouchard, David C. Bellinger, Claudio Colosio, Philippe Grandjean, Raul Harari, et al. 2012. "Neurobehavioral and Neurodevelopmental Effects of Pesticide Exposures." NeuroToxicology 33 (4) (August): 887-896. doi:10.1016/j.neuro.2012.01.004.

\section{Published Version}

doi:10.1016/j.neuro.2012.01.004

\section{Permanent link}

http://nrs.harvard.edu/urn-3:HUL.InstRepos:34767897

\section{Terms of Use}

This article was downloaded from Harvard University's DASH repository, and is made available under the terms and conditions applicable to Other Posted Material, as set forth at http:// nrs.harvard.edu/urn-3:HUL.InstRepos:dash.current.terms-of-use\#LAA

\section{Share Your Story}

The Harvard community has made this article openly available.

Please share how this access benefits you. Submit a story.

\section{Accessibility}




\title{
Neurobehavioural and neurodevelopmental effects of pesticide exposures
}

\author{
Leslie London ${ }^{1}$, Cheryl Beseler ${ }^{2}$, Maryse F. Bouchard ${ }^{3}$, David C. Bellinger ${ }^{4}$, Claudio \\ Colosio $^{5,6}$, Philippe Grandjean ${ }^{7,8}$, Raul Harari ${ }^{9}$, Tahira Kootbodien ${ }^{1}$, Hans Kromhout ${ }^{10}$, \\ Francesca Little ${ }^{11}$, Tim Meijster ${ }^{10,12}$, Angelo Moretto ${ }^{5,13}$, Diane S. Rohlman ${ }^{14}$, and Lorann \\ Stallones $^{2}$
}

${ }^{1}$ Centre for Occupational and Environmetal Health Research, School of Public Health and Family Medicine, University of Cape Town, South Africa ${ }^{2}$ Psychology Department, Colorado State University, Fort Collins, USA ${ }^{3}$ Département de santé environnementale et au travail, Université de Montréal, C.P. 6128 Succursale Centre-Ville, Montreal, CANADA ${ }^{4}$ Departments of Neurology, and Environmental Health, Harvard Medical School, Harvard School of Public Health, Boston Children's Hospital ${ }^{5}$ Department of Occupational and Environmental Health, Università degli Studi di Milano, Italy ${ }^{6}$ International Centre for Rural Health (ICRH), San Paolo Hospital, Milano, Italy ${ }^{7}$ Department of Environmental Health, Harvard School of Public Health, Boston, United States ${ }^{8}$ Department of Environmental Medicine, University of Southern Denmark, Odense, Denmark ${ }^{9}$ Corporación para el Desarrollo de la Producción y el Medio Ambiente Laboral, Quito, Ecuador ${ }^{10}$ Institute for Risk Assessment Sciences, Utrecht University, Utrecht, the Netherlands ${ }^{11}$ Department of Statistical Sciences, University of Cape Town, South Africa ${ }^{12}$ TNO, Department of Quality and Safety, Zeist, The Netherlands ${ }^{13}$ International Centre for Pesticides and Health Risk Prevention (ICPS), Luigi Sacco Hospital, Milano, Italy ${ }^{14}$ Center for Research on Occupational and Environmental Toxicology, Oregon Health and Science University, Portland, OR 97239 , USA

\section{Abstract}

The association between pesticide exposure and neurobehavioral and neurodevelopmental effects is an area of increasing concern. This symposium brought together participants to explore the neurotoxic effects of pesticides across the lifespan. Endpoints examined included neurobehavioral, affective and neurodevelopmental outcomes amongst occupational (both adolescent and adult workers) and non-occupational populations (children). The symposium discussion highlighted many challenges for researchers concerned with the prevention of neurotoxic illness due to pesticides and generated a number of directions for further research and policy interventions for the protection of human health, highlighting the importance of examining potential long-term effects across the lifespan arising from early adolescent, childhood or pre-natal exposure.

\footnotetext{
(C) 2012 Elsevier B.V. All rights reserved.

Conflict of interest: OHSU and Dr. Rohlman have a significant financial interest in Northwest Education Training and Assessment, LLC, a company that may have a commercial interest in the results of this research and technology. This potential conflict of interest was reviewed and a management plan approved by the OHSU Conflict of Interest in Research Committee was implemented.

Publisher's Disclaimer: This is a PDF file of an unedited manuscript that has been accepted for publication. As a service to our customers we are providing this early version of the manuscript. The manuscript will undergo copyediting, typesetting, and review of the resulting proof before it is published in its final citable form. Please note that during the production process errors may be discovered which could affect the content, and all legal disclaimers that apply to the journal pertain.
} 


\section{Keywords}

pesticides; neurobehavioural; neurodevelopmental; toxicity; acute poisoning; long-term exposures; injury; affective disorders

\section{Introduction}

The focus of this symposium, part of the International Neurotoxicology Association (INA) and International Commission of Occupational Health (ICOH) Joint International Neurotoxicology Conference in Xian, China, June 5-10, 2011, was to highlight some of the current challenges and advances in our understanding of the health effects of pesticide exposure across the lifetime. In featuring topics ranging from developmental toxicity studies using animal models to on-going epidemiological studies of the effects of pesticide exposure at all stages of the human life course, this symposium signaled the importance of adopting a lifecourse approach to disease epidemiology (Ben Shlomo and Kuh, 2002). Organized by Leslie London and Cheryl Beseler, the symposium, comprising 5 papers, began with a discussion of the inherent limitations in developmental toxicity testing before moving into studies of neurobehavioral deficits and Attention Deficit and Hyperactivity (ADHD) in children, neurobehavioral changes in adolescents applying organophosphate (OP) pesticides to cotton in Egypt and concluding with studies of depression and suicidality in adults with a history of pesticide poisoning and cumulative low-dose exposure to OPs.

Developmental neurotoxicity studies have been informed by the epidemiological data showing that prenatal exposure to OP pesticides may be associated with an increased risk of pervasive developmental disorders, delays in cognitive development, and attentional deficits. Postnatally, children are at greater risk from OP toxicity than adults because the brain is rapidly developing, the dose of pesticides per body weight is likely to be larger in children and children have a reduced capacity for detoxifying xenobiotics. In children, OP exposure has been associated with behavioral problems, poorer short-term memory and motor skills, and longer reaction time. Given ubiquitous exposures to OPs in the environment, the need for further toxicological and epidemiological data to characterise the nature of the risk to children is evident.

Amongst older subjects, such as adolescent and adult agricultural workers, workplace injuries and exposure to pesticides pose health hazards. Pesticides are widely used in developing countries where child labour remains a feature of many countries' agricultural sectors. High levels of exposure, including poisoning, may result in an elevated risk of agricultural injury and neuropsychiatric sequelae. Pesticide poisoning is thought to alter neurotransmitter systems in the adult brain leading to increased anxiety and depression. Further, suicide is a major public health problem in developing countries, often involving the use of pesticides as the agent for suicide. Recent work suggests that exposure to organophosphate pesticides affecting non-cholinergic systems may contribute to depression, impulsivity or some combination of these disturbances in mood and could explain an elevated association of organophosphate exposure with suicide.

Drs. Moretto and Colosio provided a starting point for the symposium with a discussion of the issues associated with developmental neurotoxicity studies currently conducted for regulatory purposes. These studies are conducted according to approved guidelines and indications for the interpretation of the results are available. Many pesticides, especially organophosphates, have been studied according to experimental protocols using varying doses and assessing different parameters. These studies were reviewed with the aim of 
identifying toxicity endpoints that may help in understanding the results of epidemiological studies.

Drs. Harari and Grandjean presented a study using an expanded battery of neurobehavioral tests in 87 children attending two grades in a local public school in northern Ecuador, where floriculture is intensive. Children with prenatal exposure from maternal greenhouse work showed consistent deficits particularly for motor speed, motor coordination, visuospatial performance and visual memory. These associations corresponded to a developmental delay equivalent to 1.5-2 years. These findings support the notion that prenatal exposure to pesticides, at levels not producing adverse health outcomes in the mother - can cause lasting adverse effects on brain development and contribute to a "silent pandemic" of developmental neurotoxicity.

Dr. Bouchard and colleagues examined the association between urinary concentrations of dialkyl phosphate metabolites of organophosphates and ADHD in children (8-15 years) representative of the general US population. They found that children with higher urinary dialkyl phosphate concentrations, especially for dimethyl alkylphosphate, were more likely to be diagnosed as having ADHD.

Dr. Rohlman and colleagues discussed their study of pesticide exposure amongst adolescents in Egypt hired seasonally to work as pesticide applicators for the cotton crop. Adolescent pesticide applicators $(n=58)$ and controls who did not apply pesticides were recruited from the same villages $(n=40)$ to participate in a 10-month longitudinal study from April 2010 to January 2011. The results showed depression in both plasma butyrylcholinesterase (PChE) and red blood cell acetylcholinesterase (AChE) levels in applicators followed by recovery after the application had ended. Applicators had impaired performance compared to controls on the majority of tests on the neurobehavioral battery developed for the study. Work practice questionnaires indicate limited use of personal protective equipment. This study provided data to characterize chlorpyrifos exposure and identified numerous behavioral deficits in an adolescent population occupationally exposed to pesticides.

Drs. Beseler and Stallones presented evidence that a history of pesticide poisoning and depression are risk factors for farm injuries. A total of 1,637 Colorado farm residents were assessed to address associations between pesticide poisoning, depressive symptoms, safety behaviors and injury in a Structural Equation Model. This work provides evidence that pesticide poisoning differentially affects the negative affect, somatic and retarded activity dimensions of depression, but not positive affect to the degree that health or financial problems do. The negative affect symptoms feeling depressed, fearful, sad and lonely and the retarded activity symptoms feeling unable to "get going" and that everything was an effort showed the most robust association with injury.

Dr. London and colleagues presented their cross-sectional study of 810 workers on commercial grape farms in the Western Cape province of South Africa showing an association between reported past poisoning by pesticides and depression as measured on the GHQ, but no relationship with long term cumulative exposure. Structural Equation Modelling did not improve the exposure-effect characterization nor identify any relevant causal pathways. Whereas this study confirmed the association of affective disorders with past poisoning consistent with the literature, the contribution of long-term low-dose exposures to depression and suicidality remains uncertain. 


\section{Evaluation of experimental data for developmental neurotoxicity of pesticides}

The development of the central nervous system occurs both in utero and postnatally, and requires an adequate environment that depends on a complex relation between different factors that have different spatial and temporal roles. Disturbances of development may have genetic as well as external factors acting during any of the phases of development (Connors et al., 2008). Many groups of pesticides act through a neurotoxic mechanism that is relevant both to target and non-target mammals, including humans. The majority of such neurotoxic compounds are included in the groups of anticholinesterases, i.e. organophoshates (OP) and carbamates, pyrethroids, and organochlorines, although other groups or individual compounds might also show neurotoxic properties. Consequently, the issue of possible effects by pesticides on the normal development of the central nervous system was raised and ways of addressing the identification and prevention of these effects have been discussed (Barlow et al., 2007; Eskenazi et al., 2008; Fitzpatrick et al., 2008; Raffaele et al., 2010). In particular, in the USA the passage in 1996 of the Food Quality Protection Act mandated an increased effort on the assessment of the potential toxicity of pesticides to children, and a special focus was given to developmental neurotoxicity (Raffaele et al., 2010).

A number of epidemiological studies have been performed to identify possible consequences on the neurological development after perinatal exposure to pesticides, and results have been subject to several criticism regarding the relevance of the findings (for a review see e.g.: Bjorling-Poulsen et al., 2008; Jurewicz and Hanke, 2008; Weselak et al., 2007). In particular, it has been concluded that many of the studies suffered from poor exposure estimation, that the effects were inconsistent, and that there was limited or inadequate evidence to support causality between neurodevelopment and perinatal low level repeated pesticide exposure. Given these uncertainties, a review of the experimental evidence was undertaken in order to assess whether animal data support the hypothesis of specific neurodevelopmental effects of pesticides; in other words the question asked was that of a particular sensitivity of the developing organism to neurotoxic effects, that occur at doses that are lower than the doses causing neurotoxic effects in the adult, including the pregnant animal.

The design of developmental neurotoxicity (DNT) studies has been the subject of specific guidelines, but there remain a number of issues related to their interpretation. Issues related to normal variability (Raffaele et al., 2008), statistics (Holson et al., 2008), use of adequate positive controls (Crofton et al., 2008), and identification and interpretation of effects (Tyl et al., 2008) have been found to be particularly relevant. Treatment-related effects can be obscured by excessive variability, or, on the other hand, minor, but statistically significant changes, can be considered as biologically significant and treatment related, when in fact they might fall within the normal range (Raffaele et al., 2008). Since DNT studies, including those not performed according to the Guidelines, generally entail a high number of comparisons and significance tests, an a priori statistical analysis that takes into account this fact is strongly suggested. When a number of the DNT studies submitted to EPA were analyzed in this respect, several inadequate approaches have been identified. These included, among others, inadequate Type I error control, power considerations, and allocation of gender, time, and litter as relevant factors in the analysis. It has been emphasized that potential p-values in a typical DNT test can amount to over 1300; a fact that with a significant $\mathrm{p}$ set at $<0.05$, leads to 65 expected significant results by chance alone (Holson et al., 2008). It is widely accepted that positive controls in DNT studies should be introduced in the experimental design (see Crofton et al., 2008 for a review) as one of the tools to demonstrate the proficiency of the performing laboratory, and also to determine the biological significance of positive results or provide confidence in negative results. Given all these problematic issues, detection, measurement and interpretation of effects are not simple 
tasks. A weight-of-evidence approach has been suggested where the qualitative nature and magnitude of effects, and their temporal patterns of development, should be taken into account. In addition, all findings should be put within the context of the study and interpreted in conjunction with other findings and other studies. Even statistically significant findings should be consistent with a pattern of effects; e.g.: dose related or accompanied by other biologically related effects (Tyl et al., 2008).

According Raffaele et al. (2010), as of December 2008, DNT studies on 72 pesticide active ingredients have been performed and submitted for review to the EPA. The study design included treatment of rat dams from gestational day 6 to post-natal day 11 or 21, the latter being the experimentally preferred one. Offspring were directly treated when exposure via maternal milk could not be demonstrated. A high number of functional, neurobehavioural, morphological and morphometric parameters were evaluated in offspring, but much fewer and much less sensitive parameters were evaluated in dams. Thus, the findings and related no/lowest-observable-adverse-effect levels (NOAELs/LOAELs) are not strictly comparable between offspring and dams, and no firm conclusion can be drawn about the relative sensitivity of dams and pups. In about $20 \%$ of the cases the NOAEL in pups or young animals was lower than in adults (in the same or other studies) and in another $20 \%$ of the cases it was likely to be so, although full information was not available in the latter case. It should be noted that the toxicity endpoints that were observed at the lowest-observableadverse-effect level (LOAEL) were generally not specifically neurotoxic (e.g. reduced body weight) and were transient (e.g. observed at an early life-stage but not later). In particular, of the 15 studies showing a lower NOAEL for pups, the effect seen at the LOAEL was reduced body weight or body weight gain, alone or more frequently accompanied by other effects in 11 studies, and reduced survival in 2 studies. Moreover, information was not available on dose spacing (i.e. between the NOAEL and the LOAEL) in DNT vs. other studies, and therefore it cannot be ascertained whether the LOAEL in the DNT study was lower than the NOAEL in other studies. Only in this case it could be concluded that the offspring were really more sensitive than adult animals. In conclusion, it appears that DNT studies performed according to regulatory guidelines may discover a higher sensitivity of animals to toxic effects when exposed perinatally. However, it remains to be ascertained in how many cases this is an artifact of dose-spacing, and/or of limited endpoint evaluations in dams. It should be noted that the NOAELs were based on effects at the LOAEL that were not neurotoxic and most of the time were associated with signs of general systemic toxicity such as those on body weight (gain). In addition, tested compounds were known neurotoxicants (mainly OPs), frequently there was a sole effect, either systemic or neurodevelopmental, and in $30 \%$ of the cases there was a sole and transient effect observed. Taken together, these data point out that pup sensitivity to neurotoxicants at the NOAEL generally is not higher, or significantly higher, than that of adult animals.

In addition to the studies performed for regulatory purposes, more studies that used different protocols and assessed different, allegedly more sensitive, endpoints have been performed with the aim to define and understand possible developmental neurotoxic effects of chemicals, in particular pesticides (see e.g.: Levin et al., 2010; Slotkin et al., 2008a; 2008b; Timofeeva et al., 2008; Torres-Sanchez et al., 2009). Most of these studies have been performed with OPs and the doses used were reported to be "low", in most cases meaning not causing overt clinical signs or even asymptomatic acetylcholinesterase (AChE) inhibition, but to be around the NOAEL for AChE inhibition. It should be pointed out that it is known that recovery from $\mathrm{AChE}$ inhibition by synthesis of new enzyme is much quicker in neonatal animals than in adults. Therefore, timing of measurement of AChE activity is most critical in younger animals, and measurements performed at e.g. $24 \mathrm{~h}$ or more after dosing, as is the case in most of these studies, may identify some inhibition in adults but miss an earlier, quickly recovered, inhibition in neonatal animals (Eaton et al., 2008). This is 
particularly relevant because it is known that acute insults to the developing brain, even after an "apparent" recovery may have long-lasting consequences (Counotte et al., 2011, Pugh et al., 2011). Scattered, and sometimes in opposite directions, biochemical, molecular and/or neurobehavioral changes were observed in these studies at "low doses", and compoundspecific (among OPs) and even sex-specific effects and mechanisms have been advocated to explain the results. While, as indicated above, most of the effects may have in fact occurred at doses causing AChE inhibition, and may be a consequence of this inhibition, no clear biological explanation could be given to those changes. Clear effects were instead observed with doses causing AChE inihibition in both adults and pups. Consequently, the results at "low doses" of these studies are of difficult interpretation for human risk assessment because changes were inconsistent both within and between studies, and of unknown or questionable biological relevance. It should also be noted that, while it is widely accepted that in many instances younger organisms are more susceptible to neurotoxic effects of relatively high single doses of chemicals, this may not be held true for repeated, lower doses (Vidair, 2004). Consequently, it is still debated whether at doses comparable to those humans are exposed to as a consequence of presence of trace contaminants in food and in the living environment, young individuals are, in fact, more sensitive than adults, and whether extrapolation of data (NOAEL/LOAEL) from pups given a bolus dose (e.g.: by gavage) to the human situation is feasible. In addition, it should be noted that while butyrylcholinesterase activity appears to have some role in the development of the nervous system (Eaton et al., 2008), it was not measured in these studies.

In conclusion, epidemiological and experimental data neither support the possible neurodevelopmental effect of pesticides at doses below the NOAEL identified in other studies (eg.: for OPs below the doses causing AChE inhibition), nor indicate a significantly higher susceptibility of young animals to repeated low doses of chemicals. Certain biochemical findings reported in several studies do not have a clear biological explanation. In this respect, it should be particularly emphasized that any attempt of testing hypotheses on the same data that generated them should be avoided and ad hoc experimental designs should be developed (Holson et al., 2008). However, as also indicated by studies with other compounds (Counotte et al., 2011), treatment of developing brain with doses of chemicals that cause reversible effects (e.g. on receptors) can have long-lasting effects appearing in adulthood. Therefore, both epidemiological and experimental studies should focus on the consequences of acute (relatively) high exposures, to understand and assess long-term effects. For these reasons it is of primary importance that public health interventions focus on prevention of such exposures to pesticides that usually derive from lack of training and/or of adequate equipment.

\section{Risks of Neurobehavioral Deficits in Children Prenatally Exposed to Pesticides in Developing Countries}

Pesticides are widely used in developing countries to control a variety of pests. Although pesticides are designed to be toxic, many of them toxic to the nervous system of insects and other species, the long-term risks from prenatal exposures to humans are unclear (BjorlingPoulsen et al. 2008). Concern has previously been raised about the agricultural use of pesticides and their possible effects on children's neurodevelopment (Guillette et al. 1998). In Ecuador, a unique situation exists on the Andean plateau. Floriculture is intensive and relies on female employment. As few other job opportunities exist, children in communities, such as Tabacundo, are therefore likely to have been exposed to pesticides from the mother's work during pregnancy.

Two cross-sectional studies of children aged 6-8 years assessed neurobehavioral functions in regard to the maternal occupational history (Grandjean et al. 2006; Harari et al. 2010). On each occasion, close to 100 children attending the two lowest grades in the local public 
school were examined. Information on pesticide exposure during the index pregnancy was obtained from maternal interview. The study design therefore aimed at comparing two groups of children (prenatally exposed versus non-exposed) with similar background, except for the maternal history of occupational exposure to pesticides. Some socioeconomic indicators showed better conditions in the exposed group, possibly due to the possibility for both parents to be economically active. Almost one-half of the children had been prenatally exposed to pesticides.

Results showed consistent deficits that were the strongest for motor speed (Finger Tapping); motor coordination (Santa Ana Form Board); visuospatial performance (Stanford-Binet Copying); and visual memory (Copying recall). These associations corresponded to a developmental delay of 1.5-2 years history (Grandjean et al. 2006; Harari et al. 2010). Prenatal pesticide exposure was also significantly associated with an average increase of 3.6 $\mathrm{mm} \mathrm{Hg}$ in systolic blood pressure, perhaps as an additional indication of developmental neurotoxicity. Joint analysis of the data from the two studies strengthened the conclusions.

Although several pesticides are used in floriculture, organophosphates may be of particular relevance in regard to our results. Acetylcholine is a major synaptic transmitter substance that also serves as a neurotrophic signal during brain development (Slotkin 2004). Experimental studies in rodents suggest that cholinesterase inhibitors can interfere with the brain development and lead to permanent damage (Slotkin 2004; Ahlbom et al. 1995). Epidemiological evidence of the neurodevelopmental toxicity of pesticide exposure during pregnancy is growing (Bjorling-Poulsen et al. 2008; Grandjean and Landrigan 2006; Gray and Lawler 2011). The results suggested that developmental pesticide exposure can cause delayed mental development, reduced motor functions and visual acuity (Handal et al. 2008), and reduced short-term memory and attention and more general cognitive deficits (Bouchard et al. 2011; Engel et al. 2011; Rauh et al. 2011).

In developing countries, effects produced by nutritional problems must be separated from those associated with pesticides (Grandjean et al. 2006). Stunting is frequently used as an objective indicator of malnutrition, and it has recently been reported in one out of every four children $<5$ years of age in Ecuador (Larrea and Kawachi 2005). In accordance with the fetal origins hypothesis (Walker et al. 2001), we found that both prenatal nutritional deficiencies and toxicant exposures may adversely affect cardiovascular development. In fact, the children with the most severe deficits were those who were stunted and also exposed prenatally to pesticides.

A study with a cross-sectional design and retrospective assessment of prenatal exposure cannot provide information about dose-response relationships or the time of the impact of the exposures. However, the standardized and blinded techniques applied to the maternal reports support the validity of the employment-based classification of exposures. The deficits associated with prenatal pesticide exposure may have long-term health consequences as part of a "silent pandemic" of developmental neurotoxicity (Grandjean and Landrigan 2006). The growing evidence on pesticides should raise attention in regard to protection of female workers during pregnancy.

For pregnant women at work, Agreement 103 of the International Labour Organization (ILO) requires two weeks before and 10 weeks after delivery to rest (see:

http://www.ilo.org/global/lang--en/index.htm). Nonetheless, the practice in Ecuador is that mothers generally prefer to work until the very last day before childbirth to allow more time with their child after birth. Improved control of exposures is needed to protect all workers and the surrounding environment and to relocate pregnant women to areas or working 
activities that do not involve exposure to pesticides. If use of pesticides cannot be avoided, appropriate personal protection must be made available and applied.

\section{Attention Deficit/Hyperactivity Disorder and Urinary Metabolites of Organophosphate Pesticides in U.S. Children 8-15 Years}

The EPA considers food, drinking water, and residential pesticide use as important sources of exposure (Cohen Hubal et al., 2000). Children are generally considered to be at greatest risk from OP toxicity because the developing brain is more susceptible to neurotoxicants, and the dose of pesticides per body weight is likely to be larger in children. Children age 611 years have the highest urinary concentrations of dialkyl phosphate (DAP) metabolites -markers of OP pesticides exposure -- compared to other age groups in the U.S. population (Barr et al., 2004). Contributing to their vulnerability, children have reduced expression of detoxifying enzymes (Holland et al., 2006). Epidemiological studies linking exposure to OP pesticides and neurodevelopment have focused on populations with high exposure relative to the general population. Prenatal exposure to OP pesticides was associated with increased risk of pervasive developmental disorders, delays in cognitive development, and attentional deficits (Rauh et al., 2006, Eskenazi et al., 2007; Bouchard et al., 2011; Marks et al., 2010; Engel et al., 2011; Rauh et al., 2011). Postnatal OP exposure has been associated with behavioral problems, poorer short-term memory and motor skills, and longer reaction time in children (Grandjean et al., 2006; Ruckhart et al., 2004; Rohlman et al., 2005). Very little data is available on OP exposure risks in children with average level of exposure. Using data on a representative sample of U.S. children, this study examined the cross-sectional association between urinary DAP metabolite concentrations and ADHD prevalence in children of ages $8-15$ years.

The National Health and Nutrition Examination Survey (NHANES 2000-2004), a population-based health survey of non-institutionalized U.S. residents, was used in this study. The Diagnostic Interview Schedule for Children (DISC-IV) was used to assess the presence of ADHD in children 8-15 years. The diagnosis is based on the presence, during the prior 12 months, of symptoms related to inattention, hyperactivity and impulsivity, with significant impairment in two or more settings (e.g., at school and home) (Shaffer et al., 2007). Six urinary DAP metabolites were measured in "spot" urine samples to provide an indicator of the body burden of common OPs in a random subsample of the participants (National Research Council, 1993). The urinary DAP metabolites measured are 3-dimethyl alkylphosphate (DMAP) molecules and 3-diethyl alkylphosphate (DEAP) molecules. We conducted statistical analysis to account for the multistage probability sampling design of NHANES and obtain robust linearized standard errors and unbiased point estimates. Urinary concentrations of DAP were log-transformed. Logistic regression analysis was used to estimate odds ratios (ORs) and 95\% confidence intervals (CI) for ADHD per 10-fold increase in DAP metabolites concentration. The following covariates were included in all models: sex, age, race/ethnicity, poverty-to-income ratio, fasting time, and log-transformed urinary creatinine. Children who received newborn care in an intensive care unit or premature nursery ( $n=167)$, those with birth weight below 2,500 grams $(n=126)$, children with extremely diluted urine (creatinine $20 \mathrm{mg} / \mathrm{dL} ; \mathrm{n}=24$ ), outlier for urinary DAP concentration $(n=1)$, and children with missing data on covariates $(n=82)$ were excluded from the analysis.

The analytic sample comprised 1,139 children 8 to 15 years. One hundred nineteen children met the diagnostic criteria for any ADHD subtype, which corresponds to a population prevalence of $12.1 \%$ (95\% CI, 9.6-15.1\%). The odds of meeting the DISC-IV criteria for ADHD increased with the urinary concentration of total DAP metabolites (Table 1). Adjustment for covariates only slightly attenuated the estimates (for a 10-fold increase in total DAP, unadjusted OR 1.3, CI1.1-1.6; adjusted OR 1.2, CI 0.97-1.5). This association 
was driven by DMAP metabolites, for which the association was statistically significant even after covariate adjustments (OR 1.6, CI 1.1-2.1). When children taking ADHDmedication were included as cases, slightly higher effect estimates were obtained for DMAP (adjusted OR 1.7, CI 1.3-2.3). DEAP metabolites were not significantly associated with odds of ADHD. The metabolite dimethylthiophosphate was the most commonly detected DMAP (64.3\% of children). Children with creatinine-adjusted dimethylthiophosphate concentrations above the median of detectable values had double the odds of ADHD compared to those with concentrations below the detection limit (adjusted OR 1.9, CI 1.23.0). The effect estimates were not affected by adjusting for blood lead concentration, maternal age at birth, or maternal smoking during pregnancy.

The odds of meeting the diagnostic criteria for hyperactive-impulsive subtype increased significantly with higher DEAP, DMAP, and total DAP (adjusted OR for a 10-fold increase in concentration 2.2, CI 1.1-4.4, 2.1, CI1.1-4.2, and 1.9, CI 1.0-3.3, respectively). The odds of inattentive subtype increased with higher concentrations of DMAP metabolites although this did not reach significance level (adjusted OR 1.5 [0.99-2.2]). Concentrations of DAP metabolites were not significantly associated with odds of combined subtype.

The study indicated an association between urinary DMAP metabolite concentrations -indicators of exposure to dimethyl-containing OP pesticides -- and increased odds of ADHD in children 8-15 years. There was a 55\% to $72 \%$ increase in the odds of ADHD for a 10-fold increase in DMAP concentration, depending on criteria used for case identification. Whether DAP metabolite concentrations are more strongly associated with a specific ADHD subtype is uncertain due to the small numbers of cases, though the association was stronger for the hyperactive-impulsive subtype. This study is generalizable to the U.S. population, unlike previous studies among highly exposed groups (Rauh et al., 2006; Eskenazi et al., 2007; Grandjean et al., 2006; Ruckhart et al., 2004; Rohlman et al., 2005).

An important limitation of the present study is the assessment of OP pesticides exposure by measurement of DAP metabolites in one spot urine sample. Long-term exposure to OP pesticides would likely be necessary to produce neurochemical changes causing ADHD-like behaviors, and serial measurements of urinary metabolites of OP pesticides over a longer time period would provide a better assessment of average exposure. For OP pesticides coming from the diet, the measure of metabolites in a single urine sample may reflect average exposure levels reasonably well, to the extent that diet is consistent. Given that OP pesticides are eliminated from the body after 3-6 days (Bradway et al., 1977), the detection of DAPs in the urine of most children indicates continuing exposure.

In conclusion, the findings support the hypothesis that current levels of OP pesticides exposure might contribute to ADHD prevalence. Future studies should employ a prospective design, with multiple urine samples collected over time to better assess chronic exposure and critical windows of exposure, and establish appropriate temporality.

\section{The impact of pestcide exposure in adolescents across an application season}

Agricultural workers, both adults and adolescents, are at risk for many occupational hazards including workplace injuries and exposure to pesticides. Experimental animal studies indicate that the developing brain is more susceptible to the neurotoxic effects of organophosphorus pesticides (OP) than the adult brain, and low-level exposures to OPs cause significant neurobehavioral deficits (Levin et al. 2001; Slotkin et al. 2001; Slotkin et al. 2002). The human adolescent brain is still developing; yet little is known about the extent or magnitude of health problems related to occupational exposure to pesticides in adolescents. Adolescents in Egypt are hired seasonally to work as pesticide applicators for 
the cotton crop. Pesticide application to the cotton crop is highly regulated and standardized across Egypt, and has been limited primarily to OPs, generally chlorpyrifos.

Behavioral studies of pesticide applicators, greenhouse workers, agricultural workers and farm residents exposed repeatedly over months or years to low levels of OPs reveal a relatively consistent pattern of neurobehavioral deficits. Although several studies have examined children who live in agricultural communities or whose parents work in agriculture, only a few studies have examined adolescents who are currently working in agriculture (Eckerman et al. 2007; Rohlman et al. 2007; Rohlman et al. 1999). The most extensive range of deficits were shown in a population of adolescents applying pesticide in Egypt (Abdel Rasoul et al. 2008). Children and adolescents (ages 9-19) who applied pesticides demonstrated impaired neurobehavioral performance, reported more symptoms, and had lower AChE levels than children from the same communities that do not apply pesticides. This study also shows a correlation between days worked during the current season and increased symptom reports and also with decreased neurobehavioral performance. However, this study is limited by the absence of exposure data.

An ongoing study is examining the impact of pesticide exposure across the application season in adolescents working as pesticide applicators, compared to controls. The goal of this project is to examine the dose-related response of the adolescent nervous system to OP pesticides, to determine if repeated exposures produce a progressive deficit and to determine if this deficit is reversible.

The Egyptian national government provides the seeds for, purchases the production of and sells the country's entire cotton production. Once farmers agree to plant cotton in their fields, applications of chemicals on those fields come under control of the Ministry of Agriculture. Each Governorate has a Deputy Minister of Agriculture who is responsible for pesticide applications on the cotton crop in their governorate. Application equipment (sprayers) and pesticides are purchased by the central Ministry office in the Governorate, the equipment is calibrated by their staff and then it is distributed to the agricultural district offices along with the pesticides to be used. Thus, all pesticides, equipment and calibration procedures are standardized across the Governorate and follow strict guidelines set out by the national Ministry of Agriculture for Governorates throughout the country. Adolescents living in villages near the fields are hired as seasonal workers to apply pesticides and may work for repeated seasons. Applicators wear backpack sprayers driven by a 2-cycle engine and apply pesticides in a staggered line through the cotton fields. Insecticides are usually applied in three waves of approximately 15 days each, according to the following pattern: OPs, pyrethroid pesticides, and OPs. The primary OP used in Egyptian cotton fields is Pestban ${ }^{\mathrm{TM}}$, an emulsifiable concentrate formulation that contains $48 \%$ chlorpyrifos as the active ingredient.

Participants included adolescents 12 to 18 years of age working as pesticide applicators $(\mathrm{N}=58)$ for the cotton crop and controls $(\mathrm{N}=40)$ from the same villages with similar age and education recruited for the study. The pesticide applicators worked in teams of 3 to 4 applicators with adult supervisors and investigators that oversaw the application and monitored the pest infestation; these teams were responsible for the pesticide application in every village in the Governorate. Information was collected from the participants on demographics, occupational history, work practices, and symptoms. Participants completed a battery of neurobehavioral tests, including both non-computerized tests and tests from the computer-based Behavioral Assessment and Research System (BARS). Urine and blood samples were collected at the beginning of the work shift. Participants completed multiple test sessions before, during and after the pesticide application season. 
The mean age of both applicators and controls was 15.5 years. Applicators reported working 4-5 days per week 5 hours per day, for an average of 3 years (range from 1-7 years). The majority of applicators (97\%) and controls (78\%) were currently enrolled in school. Because of the use of computer-based testing, previous computer use is important. Sixty-five percent of the controls and approximately half of the applicators (46\%) report using computers at least once a week. A smaller percentage of controls (8\%) compared to applicators (24\%) report never using a computer.

Applicators were asked about their use of Personal Protective Equipment (PPE) during application. Although the majority of applicators (90\%) report wearing long pants and long sleeves during application, only about half of the applicators (48\%) report wearing shoes when applying, many prefer to go barefoot when applying due to the uneven ground. None of the applicators reported wearing earplugs, gloves, safety glasses or goggles, respirators or other PPE.

Urinary metabolite levels and cholinesterase activity will be used to characterize pesticide exposure across the season. Neurobehavioral data will be used to examine behavioral outcomes associated with pesticide exposure across the season. Performance data will be associated with urinary metabolite levels and cholinesterase activity to identify the more effective biomarkers of acute and chronic exposure effects. This study will examine the dose-related response of the adolescent nervous system to OP pesticides, and provide information to determine if repeated exposures produce a progressive deficit and if this deficit is reversible.

\section{Depressive symptoms in pesticide poisoned and non-poisoned individuals: Association with injury}

Farm work is a dangerous occupation and identifying modifiable risk factors to target in injury prevention is critical. Depression is one modifiable risk factor which, if addressed, may reduce risk of injuries among farm workers (Beseler \& Stallones, 2010; Zwerling et al., 1995; Park et al., 2001; Sprince et al., 2002; Tiesman et al., 2006). One risk factor for depression is pesticide poisoning (Stallones \& Beseler, 2002; Beseler \& Stallones, 2008). Further, pesticide poisoning was related to several machine- and animal-related safety behaviors (Stallones \& Beseler, 2004; Beseler \& Stallones, 2006). Being depressed increased the probability of an injury among those who scored low on safety knowledge (Beseler \& Stallones, 2010). Thus, pesticide poisoning may result in depressive symptoms and may increase the risk of injury. This study investigated the hypothesis that specific depressive symptoms occur more often in those with a pesticide poisoning and these symptoms lead to an increased risk of farm injuries.

Study participants included principal operators and their spouses $(n=1,637)$ from two surveys conducted using similar methods. Farms (places selling \$1,000 USD or more in agricultural commodities in a normal year) were enrolled between 1993-1997; data were collected in November to March (the slow months of agricultural production in this region) of the study years. A total of 876 farm operators and their spouses representing 485 farms in Colorado were recruited and interviewed by telephone in one survey. A total of 761 principal farm operators and their spouses representing 479 farms in Northeastern Colorado were interviewed in-person at their farm by trained study personnel in the other survey.

The primary outcome was injury resulting in losing at least one day of work in the past year ( $0=$ no injury, $1=$ =injury). The 20-questions Center for Epidemiologic Studies-Depression (CES-D) was used to assess depressive symptoms. Response categories are reported based on weekly symptoms (rarely/none of the time; some of the time, occasionally, nearly all of the time); four latent constructs are represented: negative affect, somatic/retarded activity, 
positive affect and interpersonal problems (Radloff, 1977). The CES-D scale was analyzed as an ordinal variable with rarely or none of the time as the reference group. Safety knowledge was measured with a 10-item scale with possible responses being true, false, or don't know; a correct answer was coded as 0 ; incorrect/don't know responses were coded as 1. Covariates included a history of pesticide poisoning, past-year financial problems and self-perceived health. Positive responses to ever having become ill from exposure to pesticides were used to assess pesticide poisoning $(0=$ no, $1=y e s)$. An increase in debt or a decrease in income in the past year was used to assess financial problems $(0=$ no, $1=y e s)$. Self-perceived health was a binary measure $(0=$ excellent/very good/good; $1=$ fair/poor $)$. Control variables included sex (male $=0$, female $=1$ ) and age categorized into three groups $(<45,45-64,65+)$ because younger farm residents were at higher risk of injury than those in the older category and because the safety knowledge items differed by age group (Beseler \& Stallones, 2011).

Statistical analysis included descriptive statistics used to summarize the characteristics of the sample using SAS version 9.2 (The SAS Institute, Cary, NC). Item Response Theory (IRT) was used to assess CES-D item functioning and identify depressive symptoms that differed between the pesticide-poisoned and non-poisoned groups. Structural Equation Modeling (SEM) was used to model the individual depressive symptoms with significant covariates and the latent safety knowledge factor antecedent to injury. All models were run in MPlus version 5.1 (Muthén and Muthén, 2008). All tests are two-sided and results are considered significant at the $\mathrm{p}=0.05$ level of significance. Standardized coefficients are presented.

The farm sample was $53.0 \%$ male, $98.9 \%$ white, $91.4 \%$ married, $91.7 \%$ were high school graduates, fewer than $7 \%$ were in fair/poor health, $7.5 \%$ reported a past pesticide poisoning, $31.4 \%$ had financial difficulties, $7.9 \%$ were depressed by the CES-D scale and $9.4 \%$ reported an injury (154 injuries). The mean age was 48.9 (SD 13.2). The pesticide-poisoned group endorsed the most severe category of the negative affect/somatic/retarded activity symptoms at higher frequencies than the non-poisoned group (Table 2).

IRT and SEM: The positive affect items (happy, hopeful, enjoyed life, feel as good as others) did not differ between poisoned and non-poisoned farm residents (factor loadings $\mathrm{p}=0.34$; thresholds $\mathrm{p}=0.43$ ); however, thresholds (severity) were greater for negative affect items among those who were pesticide-poisoned compared to those who were not $(\mathrm{p}<0.0001)$. The best fitting SEM of injury showed that pesticide poisoning and financial and health problems preceded the negative affect construct (feeling depressed, lonely, sad, fearful, everything was an effort and could not get going), which was significantly associated with injury ( $\mathrm{p}=0.001$; Figure 1$)$.

The results suggest that negative affect/somatic/retarded activity symptoms significantly predicted injury including health and financial problems, and safety knowledge in the model. The retarded activity items feeling as if everything was an effort and that you could not "get going" are symptoms that might explain the significant associations to animal and machinery associated injuries because they require extra effort to exercise, and, importantly, they are some of the most common causes of farm injuries (Day et al., 2009). Depressed mood was a significant contributor to injury risk. It was expected that sleeping restlessly would be a significant contributor to injury risk and although it was more severe in those with a history of pesticide poisoning, it did not add to the fit of the SEM.

This work provides evidence that pesticide poisoning differentially affects the negative affect, somatic and retarded activity dimensions of depression, but not positive affect to the degree that health or financial problems do. The negative affect symptoms feeling depressed, fearful, sad and lonely and the retarded activity symptoms feeling unable to "get 
going" and that everything was an effort showed the most robust association with injury. Future work on farm injuries should target specific symptoms of depression and injury for use in behavioral health screening.

\section{The association of suicidality, depression and organosphosphate exposure amongst farm workers in South Africa}

South Africa remains the largest market for pesticides in sub-Saharan Africa, where chemical agents are widely used for control of pests in agriculture and for purposes of vector control in public health. The Western Cape Province is an intensive fruit, grape and wheat farming region, contributing close to half of South Africa's agricultural exports in 2011. However, methods to reduce dependence on chemical control of pesticides, such as Integrated Pest Management (IPM) have achieved limited impact on chemical exposures in agriculture in the region, as have other methods of exposure reduction, such as use of protective clothing. Moreover, agricultural workers in South Africa, despite enjoying formal protection under the law, remain particularly vulnerable to workplace hazards due to a combination of social factors such as low education, high alcohol consumption and highly dependent social positioning (London et al, 2011).

While the literature is relatively clear on the central and peripheral nervous system consequences of acute intoxication by pesticides, particularly organophosphate insecticides, evidence for long-term nervous system sequelae of cumulative low-dose exposures is less clear. Where such evidence is evident, the literature points to primarily symptom-based associations, often in the area of affective consequence of long-term pesticide exposures (Kamel and Hoppin, 2004). At the same time, it is well recognised that suicide is a major public health problem in developing countries, often involving the use of pesticides as the agent for suicide. Recent work suggests that a different pathway, in which exposure to organophosphate pesticides affecting non-cholinergic systems may contribute to depression, impulsivity or some combination of these disturbances in mood, could explain an elevated association of organophosphate exposure with suicide. For example, animal studies have linked OP exposure to changes in serotoninergic systems and serotonin disturbance is well known to be associated with depression. Other data supporting a possible association include cases series, as well as ecological, case control and cohort studies linking OP exposure and affective disorders (London et al, 2005), though the evidence supporting an association with suicide emerging from some of the cohort studies is equivocal.

This paper reports on a preliminary analysis of data from a cross-sectional study in 2002 involving 817 adult farm workers recruited using single stage cluster sampling from 57 commercial grape farms in the Western Cape province of South Africa, principally to explore possible causal pathways between organophosphate exposure and depression, impulsivity, suicide ideation and eventually suicide.

Participants were screened for depression, impulsivity and suicide ideation using the 28 item General Health Questionnaire (GHQ-28) and the GHQ Depression Subscale; the Brief Symptom Inventory (BSI) Global Severity Index and BSI Depression Symptom Dimension; Barratt's Impulsivity Scale (BIS-11) and Beck's Scale for Suicidal Ideation (SSI). Exposure to OP pesticides was based on a detailed occupational history exploring high exposure job tasks in current and previous jobs, as well as a and history of past pesticide poisoning. Cumulative exposure was constructed from total years worked in agriculture as well as total years worked in tasks determined a priori to be high exposure activities. Confounders examined include medical and psychiatric history, alcohol consumption (measured as current versus not current, and in terms of CAGE score), demography (age, gender, socioeconomic status and farm type) and use of protective clothing. 
SPSS Version 15.0 was used to generate descriptive statistics to summarize the characteristics of the sample, to conduct bivariate comparisons to explore gender differences in exposure histories and for multiple logistic regression analyses to examine associations between different exposure metrics and the different depression and suicide outcomes, controlled for identified confounders and effect modifiers.

As a method suited to explaining relationships among multiple variables in which hypotheses about relations among observed and latent variables may be examined (Hair et al, 1999; Hoyle, 1995), Structural Equation Modelling (SEM) was used to explore possible possible causal pathways between OP exposure and depression, impulsive behaviour and suicidal ideation. The SEM analyses used the robust maximum likelihood method due to multivariate non-normality of the data. Criteria for goodness of fit indices include a low chisquare $\left(\chi^{2}\right)$ with a non-significant $p$-value (i.e. $\mathrm{p}>0.05$ ), $\chi^{2} / \mathrm{df}<3$ and RMSEA $<0.08$. LISREL 8.8 (Scientific Statistical Software, 2007) was used for all SEM analyses.

The response rate achieved was $90 \%$ of approached farms and $61 \%$ of workers on the responding farms. After exclusion of subjects with missing data for purposes of SEM $(\mathrm{n}=65)$, the sample was reduced to 752 workers of whom $59 \%$ were male with a median age was 33.3 yrs. A history of past psychiatric illness (6\% men; $15 \%$ women) and of past pesticide poisoning ( $15 \%$ men; $11 \%$ women) was reported in a minority of respondents. However, problem drinking, as measured by a CAGE score of 2 or more, was very common among respondents (79\% men; 69\% women). Multivariate regression modeling, using as outcome different depression, impulsivity and suicide scores, dichotomized around either recommended cut offs (e.g. 'caseness' on the GHQ was defined as $\geq 24$ versus $\mathcal{2} 3$ ), or across the $75^{\text {th }}$ percentile for scores where no standard cut-offs were available, found no significant associations with cumulative exposure as expressed as cumulative years worked in agriculture or in specific high risk activities. However, past poisoning as a measure of high OP exposure, when controlled for gender, age, farm type, CAGE score, psychiatric history, PPE use, socio-economic status and cholinesterase category, was significantly associated with high score on the GHQ Severe Depression domain (OR $=1.62 ; 95 \%$ CI 1.00 $-2.63)$.

In the SEM analyses, evaluation of all measurement models demonstrated adequate goodness of fit $\left(a^{2} / d f<3\right.$; RMSEA $<0.08$; CFI and NNFI $\left.<0.90\right)$ and all measured variables loaded significantly on the respective latent constructs, with an absence of Heywood cases, indicating that all models examined were valid for structural path mapping.

Across several models for long-term exposure, there was no significant association found between long-term cumulative OP exposure and impulsivity, depression or suicide among the various models. In the modelling postulating a pathway from exposure through both impulsivity and depression to suicide, a history of past pesticide poisoning significantly and positively influenced depression on the GHQ $(\beta=0.487, p<0.001)$ and Socio-Economic Status inversely influenced impulsivity $(\beta=-0.502, \mathrm{p}<0.001)$. Both depression and impulsivity were found to map onto suicidality $(\beta=0.387 ; 0<0.05$ and $\beta=0.373 ; p<0.001$, respectively).

The findings in this exploratory analysis of the associations involving SES and past poisoning with impulsivity and depression are consistent with patterns described previously in the literature, as are the associations of depression and impulsivity with suicidality. However, the results do not provide evidence for a role for cumulative low-dose exposure to OP's. Limitations that may explain this absence of an association include the cross-sectional nature of the study design and its inherent vulnerability to the Healthy Worker Effect, weaknesses in the quality of exposure assessment and the presence in this population of very 
high levels of problem drinking, which may mask the exposure-effect relationships associated with pesticides.

In conclusion, use of Structural Equation Modelling to explore the data set did not appear to improve the characterisation of the association between cumulative OP exposure and adverse effects nor identify any relevant causal pathways involving low level exposure. Whereas the findings confirmed the association of affective disorders with past poisoning consistent with the literature, the contribution of long-term low-dose exposures to depression and suicidality remains uncertain. Further analyses of different models for possible causal pathways are underway. Additionally, studies based on longitudinal data and with prospectively collected more detailed and accurate exposure measures (including spraying schemes and info on re-entry exposure), preferably validated through biomarkers of exposure, are desirable.

\section{Conclusions}

Pesticide exposures have been associated with prenatal and postnatal effects in humans as a result of environmental exposures, as well as neurological and neuropsychiatric effects on adults who are occupationally exposed to high levels of pesticides. Concerns are greatest in developing countries where the use of PPE is cost prohibitive and often neither available nor practical. Given the association of organophosphates with behavioral problems in children, the rates of which have risen sharply in recent years, and the unknown but long-lasting effects on the developing brain, reducing exposure is critical. The toxicological database suggests that epidemiological and experimental studies should focus on the consequences of acute (relatively) high exposures, to understand and assess long-term effects. Further research should explore the biochemical basis for alterations in neurotransmitter systems so that primary care physicians and mental health professionals will have a better understanding of how to treat children and adults who have been exposed to pesticides. The presentations in this symposium signal the need to rethink potential long-term effects across the lifespan arising from early adolescent, childhood or pre-natal exposure.

\section{Acknowledgments}

The study by Rohlman et al, was supported by the National Institute of Environmental Health Sciences (NIEHS, R21 ES017223). The content is solely the author's responsibility and does not necessarily represent official views of NIEHS. Appreciation is extended to James R. Olson, Ahmed Ismail, Olfat Hendy and Gaafar Abdel Rasoul, members of the research teams on the grant that supported this work who contributed to the development of the information in this publication.

The study by Bouchard and Bellinger is based on work conducted as part of a larger team. Colleagues Robert O. Wright from the Department of Pediatrics, Harvard Medical School and Marc G. Weisskopf from Departments of Environmental Health, and Epidemiology, Harvard School of Public Health, are acknowledged for their contributions.

The study by Beseler and Stallones was supported by funding from the Centers for Disease Control and Prevention (CDC) National Institute of Occupational Safety and Health and the CDC the National Center for Injury Prevention and Control (grant number U04/CCU806060 and R49/CE001168). Its contents are solely the responsibility of the authors and do not necessarily represent the official views of the Centers for Disease Control and Prevention.

Funding for study by London and colleagues was provided by the National Research Foundation of South Africa and the South Africa-Netherlands Programme for Alternative Development. The contributions of colleagues, Viveca Major, who was responsible for overseeing data collection and primary analysis, and Alan Flisher who contributed to conceptualising the SEM design, but who died tragically before publication, are acknowledged. The findings are solely the responsibility of the authors and do not represent the views of either of the funding organisations. 


\section{References}

Abdel Rasoul GM, Abou Salem ME, Mechael AA, Hendy OM, Rohlman DS, Ismail AA. Effects of occupational exposure on children applying pesticides. Neurotoxicology. 2008; 29:833-838. [PubMed: 18662718]

Ahlbom J, Fredriksson A, Eriksson P. Exposure to an organophosphate (DFP) during a defined period in neonatal life induces permanent changes in brain muscarinic receptors and behaviour in adult mice. Brain Res. 1995; 677:13-19. [PubMed: 7606457]

Barlow BK, Cory-Slechta DA, Richfield EK, Thiruchelvam M. The gestational environment and Parkinson's disease: evidence for neurodevelopmental origins of a neurodegenerative disorder. Reprod Toxicol. 2007; 23:457-470. [PubMed: 17350799]

Barr DB, Bravo R, Weerasekera G, Cattabiano LM, Whitehead RD Jr, Olsson AO, et al. Concentrations of dialkyl phosphate metabolites of organophosphorus pesticides in the U.S. population. Environ Health Perspect. 2004; 112:186-200. [PubMed: 14754573]

Ben Shlomo Y, Kuh D. A life course approach to chronic disease epidemiology: conceptual models, empirical challenges and interdisciplinary perspectives. Int J Epi. 2002; 31:285-293.

Beseler CL, Stallones L. A cohort study of pesticide poisoning and depression in Colorado farm residents. Ann Epidemiol. 2008; 18:768-774. [PubMed: 18693039]

Beseler CL, Stallones L. Structural equation modeling of the relationships between pesticide poisoning, depressive symptoms and safety behaviors among Colorado farm residents. J Agromed. 2006; 11:35-46.

Beseler CL, Stallones L. Safety knowledge, safety behaviors depression and injuries in Colorado farm residents. Am J Industrial Med. 2010; 53:47-54.

Beseler CL, Stallones L. An item response theory analysis of safety knowledge in Colorado farm residents. J Occup Environ Med. 2011; 53:388-95. [PubMed: 21407101]

Bjorling-Poulsen M, Andersen HR, Grandjean P. Potential developmental neurotoxicity of pesticides used in Europe. Environ Health. 2008; 7:50. [PubMed: 18945337]

Bouchard MF, Chevrier J, Harley KG, Kogut K, Vedar M, Calderon N, et al. Prenatal exposure to organophosphate pesticides and IQ in 7-year-old children. Environ Health Perspect. 2011; 119:1189-1195. [PubMed: 21507776]

Bradway DE, Shafik TM, Lores EM. Comparison of cholinesterase activity, residue levels, and urinary metabolite excretion of rats exposed to organophosphorus pesticides. J Agric Food Chem. 1977; 25:1353-1358. [PubMed: 72085]

Cohen Hubal EA, Sheldon LS, Burke JM, McCurdy TR, Berry MR, Rigas MI, et al. Children's exposure assessment: a review of factors influencing Children's exposure, and the data available to characterize and assess that exposure. Environ Health Perspect. 2000; 108:475-86. [PubMed: 10856019]

Connors SL, Levitt P, Matthews SG, Slotkin TA, Johnston MV, Kinney HC, et al. Fetal mechanisms in neurodevelopmental disorders. Pediatr Neurol. 2008; 38:163-176. [PubMed: 18279750]

Counotte D, Goriounova N, Li KW, Loos M, van de Schors RC, Schettlers D, et al. Lasting synaptic changes underlie attention deficits caused by nicotine exposure during adolescence. Nat Neurosci. 2011; 14:417-419. [PubMed: 21336271]

Crofton KM, Foss JA, Hass U, Jensen KF, Levin ED, Parker SP. Undertaking positive control studies as part of developmental neurotoxicity testing. A report from the ILSI Research Foundation/Risk Science Institute expert working group on neurodevelopmental endpoints. 2008; 30:266-287.

Day L, Voaklander D, Sim M, Wolfe R, Langley J, Dosman J, et al. Risk factors for work related injury among male farmers. Occup Environ Med. 2009; 66:312-318. [PubMed: 19095702]

Eaton DL, Daroff RB, Autrup H, Bridges J, Buffler P, Costa LG, et al. Review of the toxicology of chlorpyrifos with an emphasis on human exposure and neurodevelopment. Crit Rev Toxicol. 2008; 38(Suppl 2):1-125. [PubMed: 18726789]

Eckerman DA, Gimenes LS, de Souza RC, Galvao PR, Sarcinelli PN, Chrisman JR. Age related effects of pesticide exposure on neurobehavioral performance of adolescent farmworkers in Brazil. Neurotoxicol Teratol. 2007; 29:164-75. [PubMed: 17123781] 
Engel SM, Wetmur J, Chen J, Zhu C, Barr DB, Canfield RL, et al. Prenatal exposure to organophosphates, paraoxonase 1, and cognitive development in childhood. Environ Health Perspect. 2011; 119:1182-1188. [PubMed: 21507778]

Eskenazi B, Rosas L, Marks A, Bradman A, Harley K, Holland N, et al. Pesticide toxicity and the developing brain. Basic Clin Pharmacol Toxicol. 2008; 102:228-36. [PubMed: 18226078]

Eskenazi B, Marks AR, Bradman A, Harley K, Barr DB, Johnson C, et al. Organophosphate pesticide exposure and neurodevelopment in young Mexican-American children. Environ Health Perspect. 2007; 115:792-8. [PubMed: 17520070]

Fitzpatrick J, Mendez E, Walls I. Introduction to the ILSI Research Foundation/Risk Science Institute reports from the expert working group on neurodevelopmental endpoints. Neurotoxicol Teratol. 2008; 30:263-265. [PubMed: 18407461]

Grandjean P, Harari R, Barr DB, Debes F. Pesticide exposure and stunting as independent predictors of neurobehavioral deficits in Ecuadorian school children. Pediatrics. 2006; 117:e546-556. [PubMed: 16510633]

Grandjean P, Landrigan PJ. Developmental neurotoxicity of industrial chemicals. Lancet. 2006; 368:2167-2178. [PubMed: 17174709]

Gray K, Lawler CP. Strength in numbers: three separate studies link in utero organophosphate pesticide exposure and cognitive development. Environ Health Perspect. 2011; 119:A323.

Guillette EA, Meza MM, Aquilar MG, Soto AD, Garcia IE. An anthropological approach to the evaluation of preschool children exposed to pesticides in Mexico. Environ Health Perspect. 1998; 106:347-353. [PubMed: 9618351]

Hair, JF.; Black, WC.; Babin, BJ., et al. Multivariate data analysis: A global perspective. Seventh. Pearson Prentice Hall Publishing; Upper Saddle River, New Jersey: 2011.

Handal AJ, Harlow SD, Breilh J, Lozoff B. Occupational exposure to pesticides during pregnancy and neurobehavioral development of infants and toddlers. Epidemiology. 2008; 19:851-859. [PubMed: 18813021]

Harari R, Julvez J, Murata K, Barr D, Bellinger DC, Debes F, et al. Neurobehavioral deficits and increased blood pressure in school-age children prenatally exposed to pesticides. Environ Health Perspect. 2010; 118:890-896. [PubMed: 20185383]

Holland N, Furlong C, Bastaki M, Richter R, Bradman A, Huen K, et al. Paraoxonase polymorphisms, haplotypes, and enzyme activity in Latino mothers and newborns. Environ Health Perspect. 2006; 114:985-91. [PubMed: 16835048]

Holson R, Freshwater L, Maurissen J, Moser VC, Phang W. Statistical issues and techniques appropriate for developmental neurotoxicity testing. A report from the ILSI research foundation/ risk science institute expert working group on neurodevelopmental endpoints. Neurotoxicol Teratol. 2008; 30:326-348. [PubMed: 17681748]

Hoyle, RH. Structural Equation Modelling: Concepts, issues and applications. Sage Publications Inc.; Thousand Oaks: 1995.

Jurewicz J, Hanke W. Prenatal and childhood exposure to pesticides and neurobehavioral development: review of epidemiological studies. International Journal of Occupational Medicine and Environmental Health. 2008; 21:121-32. [PubMed: 18614459]

Kamel F, Hoppin JA. Association of pesticide exposure with neurologic dysfunction and disease. Environ Health Perspect. 2004; 112:950-958. [PubMed: 15198914]

Larrea C, Kawachi I. Does economic inequality affect child malnutrition? The case of Ecuador. Soc Sci Med. 2005; 60:165-78. [PubMed: 15482876]

Levin ED, Addy N, Nakajia A, Christopher NC, Seidler FJ, Soltkin TA. Persistent behavioral consequences of neonatal chlorpyrifos exposure in rats. Brain Res Dev Brain Res. 2001; 130:8389.

Levin ED, Timofeeva OA, Yang L, Petro 'a,Ryde IT, Wrench N, et al. Early postnatal parathion exposure in rats causes sex-selective cognitive impairment and neurotransmitter defects which emerge in aging. Behav Brain Res. 2010; 208:319-327. [PubMed: 20015457]

London L, Flisher AJ, Wesseling C, Mergler D, Kromhout H. Suicide and exposure to organophosphate insecticides: cause or effect? Am J Ind Med. 2005; 47:308-321. [PubMed: 15776467] 
London, L.; Joshi, TK.; Cairncross, E., et al. Environmental Justice: an international perspective. In: Nriagu, JO., editor. Encyclopedia of Environmental Health. Vol. 2. Burlington: Elsevier; 2011. p. 441-48.

Marks AR, Harley K, Bradman A, Kogut K, Barr DB, Johnson C, et al. Organophosphate pesticide exposure and attention in young Mexican-American children: the CHAMACOS study. Environ Health Perspect. 2010; 118:1768-74. [PubMed: 21126939]

Muthén, BO.; Muthén, LK. Mplus: Statistical analysis with latent variables (version 5.1). Muthén \& Muthén Inc.; Los Angeles, CA: 2008.

National Research Council (U.S.). Pesticides in the diets of infants and children. Vol. xv. Washington, D.C.: National Academy Press; 1993. Committee on Pesticides in the Diets of Infants and Children; p. 386

Park H, Sprince NL, Lewis MQ, Burmeister LF, Whitten PS, Zwerling C. Risk factors for workrelated injury among male farmers in Iowa: a prospective cohort study. J Occup Environ Med. 2001; 43:542-547. [PubMed: 11411326]

Pugh P, Adlaf E, Zhao CS, Markwardt S, Gavin C, Wadiche J, et al. Enhanced integration of newborn neurons after neonatal insults. Front Neurosci. 2011; 5:45, 1-11. [PubMed: 21490706]

Radloff LS. The CES-D Scale: A self-report depression scale for research in the general population. Appl Psychol Measurement. 1977; 1:385-401.

Raffaele KC, Fisher JE Jr, Handcock S, Hazelden K, Sobrian SK. Determining normal variability in a developmental neurotoxicity test. A report from the ILSI Research Foundation/Risk Science Institute expert working group on neurodevelopmental endpoints. Neurotoxicol Teratol. 2008; 30:288-325. [PubMed: 18280700]

Raffaele KC, Rowland J, May B, Makris SL, Schumacher K, Scarano LJ. The use of developmental neurotoxicity data in pesticide risk assessments. Neurotoxicol Teratol. 2010; 32:563-572. [PubMed: 20398750]

Rauh VA, Garfinkel R, Perera FP, Andrews HF, Hoepner L, Barr DB, et al. Impact of prenatal chlorpyrifos exposure on neurodevelopment in the first 3 years of life among inner-city children. Pediatrics. 2006; 118:e1845-1859. [PubMed: 17116700]

Rauh V, Arunajadai S, Horton M, Perera F, Hoepner L, Barr DB, et al. Seven-year neurodevelopmental scores and prenatal exposure to chlorpyrifos, a common agricultural pesticide. Environ Health Perspect. 2011; 119:1196-1201. [PubMed: 21507777]

Rohlman DS, Lasarev M, Anger WK, Scherer J, Stupfel J, McCauley L. Neurobehavioral performance of adult and adolescent agricultural workers. Neurotoxicology. 2007; 28:374-380. [PubMed: 17141876]

Rohlman, DS.; Bailey, SR.; Anger, WK., et al. Proceedings of the Oregon Academy of Science. Salem, OR: 1999. Computerized tests to assess neurobehavioral function in Hispanic adolescents working in agriculture.

Rohlman DS, Arcury TA, Quandt SA, Lasarev M, Rothlein J, Travers R, et al. Neurobehavioral performance in preschool children from agricultural and non-agricultural communities in Oregon and North Carolina. Neurotoxicology. 2005; 26:589-598. [PubMed: 16112324]

Ruckart PZ, Kakolewski K, Bove FJ, Kaye WE. Long-term neurobehavioral health effects of methyl parathion exposure in children in Mississippi and Ohio. Environ Health Perspect. 2004; 112:4651. [PubMed: 14698930]

Shaffer, D., et al. Scoring manual: Diagnostic interview schedule for children (DISC-IV). New York: New York State Psychiatric Institute, Columbia University; 2007.

Slotkin TA. Cholinergic systems in brain development and disruption by neurotoxicants: nicotine, environmental tobacco smoke, organophosphates. Toxicol Appl Pharmacol. 2004; 198:132-151. [PubMed: 15236950]

Slotkin TA, Cousins MM, Tate CA, Seidler FJ. Persistent cholinergic presynaptic deficits after neonatal chlorpyrifos exposure. Brain Res. 2001; 902:229-243. [PubMed: 11384617]

Slotkin TA, Tate CA, Cousins MM, Seidler FJ. Functional alterations in CNS catecholamine systems in adolescence and adulthood after neonatal chlorpyrifos exposure. Brain Res Dev Brain Res. 2002; 133:163-173. 
Slotkin T, Bodwell B, Levin E, Seidler FJ. Neonatal exposure to low doses of diazinon: long-term effects on neural cell development and acetylcholine systems. Environ Health Perspect. 2008a; 116:340-348. [PubMed: 18335101]

Slotkin T, Bodwell B, Ryde I, Levin E, Seidler F. Exposure of neonatal rats to parathion elicits sexselective impairment of acetylcholine systems in brain regions during adolescence and adulthood. Environ Health Perspect. 2008b; 116:1308-1314. [PubMed: 18941570]

Sprince NL, Park H, Zwerling C, Lynch CF, Whitten PA, Thu K, et al. Risk factors for machineryrelated injury among Iowa farmers: a case-control study nested in the agricultural health study. Int J Occup Med Environ Health. 2002; 8:332-338.

Stallones L, Beseler C. Pesticide poisoning and depressive symptoms among farm residents. Ann Epidemiol. 2002; 12:389-94. [PubMed: 12160597]

Stallones L, Beseler C. Safety practices and depression among farm residents. Ann Epidemiol. 2004; 14:571-78. [PubMed: 15350957]

Tiesman HM, Peek-Asa C, Whitten P, Sprince NL, Stromquist A, Zwerling C. Depressive symptoms as a risk factor for unintentional injury: a cohort study in a rural county. Injury Prevention. 2006; 12:172-77. [PubMed: 16751447]

Timofeeva O, Sanders D, Seemann K, Yang L, Hermanson D, Regenbogen S, et al. Persistent behavioural alterations in rats neonatally exposed to low doses of the organophosphate pesticide, parathion. Brain Res Bull. 2008; 77:404-411. [PubMed: 18817854]

Torres-Sànchez L, Schanaas L, Cebrian EM, Hernandez Mdel C, Valencia EO, Garcia Hernandez RM, et al. Prenatal dichlorodiphenyldichloroethylene (DDE) exposure and neurodevelopment: a followup from 12 to 30 months of age. Neurotoxicology. 2009; 30:1162-1165. [PubMed: 19733589]

Tyl R, Crofton K, Moretto A, Moser V, Sheets LP, Sobotka TJ. Identification and interpretation of development neurotoxicity effects. A report from the ILSI Research Foundation/Risk Science Institute expert working group on neurodevelopmental endpoints. Neurotoxicol Teratol. 2008; 30:349-381. [PubMed: 17826946]

Vidair C. Age dependence of organophosphate and carbamate neurotoxicity I the postnatal rat: extrapolation to the human. Toxicol Appl Pharmacol. 2004; 196:287-302. [PubMed: 15081274]

Weselak M, Arbuckle T, Foster W. Pesticide exposure and developmental outcomes: the epidemiological evidence. J Toxicol Environ Health. 2007; 10:41-80.

Walker SP, Gaskin P, Powell CA, Bennett FI, Forrester TE, Grantham-McGregor S. The effects of birth weight and postnatal linear growth retardation on blood pressure at age 11-12 years. J Epidemiol Community Health. 2001; 55:394-398. [PubMed: 11350995]

Zwerling C, Sprince NL, Wallace RB, Davis CS, Whitten PS, Heeringa SG. Occupational injuries among agricultural workers 51 to 61 years old: a national study. J Agric Saf Health. 1995; 1:273281. 


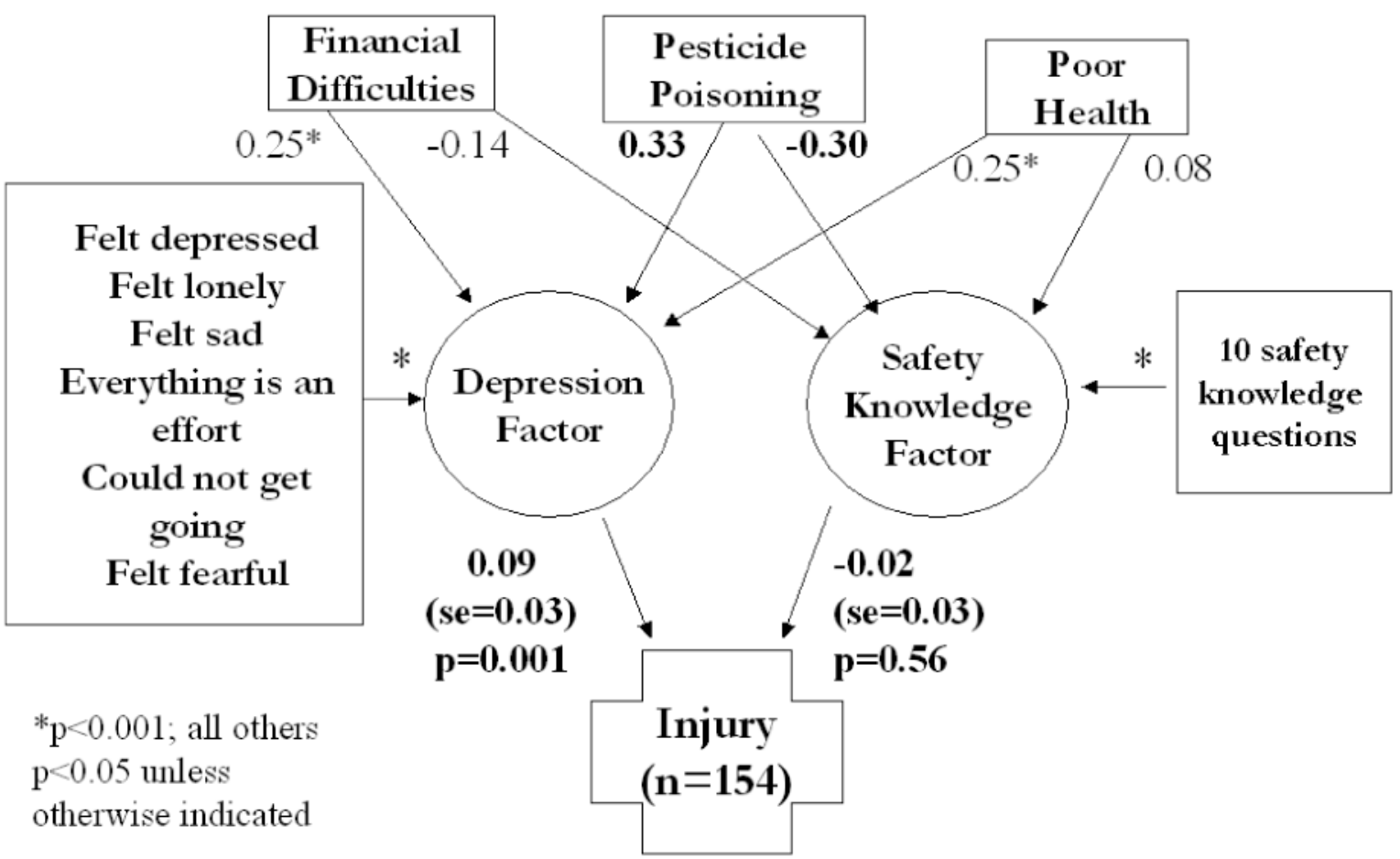

Figure 1.

Structural Equation Model of the effects of significant covariates on the latent depression dimensions of the CES-D scale, the latent safety knowledge factor and injury 
Table 1

Odds ratios (95\% confidence intervals) for any ADHD subtype for a 10-fold increase in urinary DAP metabolites

\begin{tabular}{|c|c|c|c|c|}
\hline & \multicolumn{2}{|c|}{ Cases identified with the DISC-IV ( $n=119)$} & \multicolumn{2}{|c|}{ Cases identified with the DISC-IV or ADHD-medicated $(n=148)$} \\
\hline & $\begin{array}{l}\text { Crude OR } \\
\text { (CI) }\end{array}$ & $\underset{\text { (CI) }}{\text { Adjusted }} \mathrm{OR}^{I}$ & $\begin{array}{l}\text { Crude OR } \\
\text { (CI) }\end{array}$ & $\underset{\text { (CI) }}{\text { Adjusted }} \mathrm{OR}^{1}$ \\
\hline DEAP & $\begin{array}{c}1.02 \\
(0.74-1.41)\end{array}$ & $\begin{array}{c}0.94 \\
(0.69-1.28)\end{array}$ & $\begin{array}{c}0.88 \\
(0.66-1.18)\end{array}$ & $\begin{array}{c}0.80 \\
(0.60-1.05)\end{array}$ \\
\hline DMAP & $\begin{array}{c}1.66 \\
(1.24-2.22)\end{array}$ & $\begin{array}{c}1.55 \\
(1.14-2.10)\end{array}$ & $\begin{array}{c}1.87 \\
(1.42-2.47)\end{array}$ & $\begin{array}{c}1.72 \\
(1.31-2.28)\end{array}$ \\
\hline Total DAP & $\begin{array}{c}1.31 \\
(1.06-1.63)\end{array}$ & $\begin{array}{c}1.21 \\
(0.97-1.51)\end{array}$ & $\begin{array}{c}1.48 \\
(1.20-1.82)\end{array}$ & $\begin{array}{c}1.35 \\
(1.10-1.67)\end{array}$ \\
\hline
\end{tabular}

Abbreviations: ADHD, attention deficit/hyperactivity disorder; CI, confidence interval; OR, odds ratio; DEAP, diethyl alkylphosphate; DMAP, dimethyl alkylphosphate; DAP, dialkyl phosphate.

${ }^{1}$ Sex, age, race/ethnicity, poverty-income ratio, fasting duration, and log-transformed urinary creatinine concentration 


\section{Table 2}

Frequency of experiencing a depressive symptom 5-7 days per week in the past week in those with and without a history of pesticide poisoning in 1,633 Colorado farm residents, 1993-1997.

\begin{tabular}{|c|c|c|}
\hline Depressive Symptom occurring 5 to 7 days in the past week & Percent in poisoned group & Percent in non-poisoned group \\
\hline \multicolumn{3}{|c|}{ Somatic/Retarded Activity Symptoms } \\
\hline Bothered by things that do not usually bother you & 5.0 & 2.5 \\
\hline No appetite & 4.1 & 1.5 \\
\hline Trouble concentrating & 8.3 & 4.2 \\
\hline Felt as if everything was an effort & 18.3 & 6.6 \\
\hline Slept restlessly & 11.8 & 5.7 \\
\hline Talked less than usual & 1.7 & 2.4 \\
\hline Could not "get going" & 7.6 & 2.2 \\
\hline \multicolumn{3}{|c|}{ Negative Affect Symptoms } \\
\hline Felt blue & 3.3 & 1.4 \\
\hline Felt depressed & 4.2 & 1.4 \\
\hline Felt like life had been a failure & 1.7 & 0.5 \\
\hline Felt fearful & 1.7 & 1.3 \\
\hline Felt lonely & 0.8 & 1.0 \\
\hline Felt sad & 2.5 & 0.7 \\
\hline
\end{tabular}

